\title{
Evaluation of copper alloys for reducing infection by methicillin resistant Staphylococcus aureus and vancomycin resistant Enterococcus faecium in intensive care unit and in vitro
}

\author{
Sung Im Choi ${ }^{1,{ }^{*}}$, Mee Soo Chang ${ }^{2,{ }^{*}}$, Taeeun $\mathrm{Kim}^{3}$, Kyung Hwa Chung ${ }^{3}$, Seongman Bae ${ }^{4}$, Sung-Han Kim ${ }^{4}$, \\ Chan Jin Yoon ${ }^{4}$, Young Kyoon $\mathrm{Kim}^{5}$, and Jun Hee Woo
}

\begin{abstract}
${ }^{1}$ Department of Internal Medicine, Dongguk University Ilsan Hospital, Goyang; ${ }^{2}$ Department of Pathology, Seoul National University Boramae Hospital, Seoul National University College of Medicine, Seoul;

${ }^{3}$ Department of Infectious Diseases, Uijeongbu Eulji Medical Center, Eulji University, Uijeongbu; ${ }^{4}$ Department of Infectious Diseases, Asan Medical Center, University of Ulsan College of Medicine, Seoul; ' 5 Department of Internal Medicine, Seoul St. Mary's Hospital, College of Medicine, The Catholic University of Korea, Seoul, Korea
\end{abstract}

Received: December 3, 2020 Revised : February 18, 2021 Accepted: March 25, 2021

\author{
Correspondence to \\ Jun Hee Woo, M.D. \\ Department of Infectious \\ Diseases, Uijeongbu Eulji \\ Medical Center, Eulji University, \\ 712 Dongil-ro, Uijeongbu 11759, \\ Korea \\ Tel: +82-31-951-1805 \\ Fax: +82-31-951-3300 \\ E-mail: yeom3477@daum.net \\ https://orcid.org/0000-0003- \\ 4512-5352 \\ *These authors contributed \\ equally to this work.
}

Background/Aims: Multi-drug resistant pathogens are increasing among healthcare-associated infections. It is well known that copper and copper alloys have antimicrobial activity. We evaluated the activity of copper against bacteria in a hospital setting in Korea.

Methods: This study was conducted in a laboratory and medical intensive care unit (ICU). Methicillin resistant Staphylococcus aureus (MRSA) and vancomycin resistant Enterococcus faecium (VRE) were inoculated onto copper, copper alloy and stainless steel plates. After 24 hours of incubation, colony-forming units (CFU) were counted in the laboratory. Two similar rooms were chosen in the ICU; one room had copper-containing surface, and the other room contained items with a stainless steel surfaces. Items were sampled weekly for 8 weeks when the rooms were not crowded and when the rooms were busier with healthcare workers or visitors.

Results: In vitro time-kill curves showed copper or, a copper alloy yielded a significant reduction in MRSA and VRE CFUs over 15 minutes. Upon exposure to stainless steel plates, CFUs were slowly reduced for 24 hours. In vivo, MRSA CFUs were lower in rooms with copper-containing surfaces compared with controls, both after cleaning and after patients had received visitors $(p<0.05)$. Analysis of VRE revealed similar results, but VRE CFUs from copper-containing surfaces of drug carts in the ICU did not decrease significantly.

Conclusions: Copper has antimicrobial activity and appears to reduce the number of multi-drug resistant microorganisms in a hospital environment. This finding suggests the potential of the use of copper fittings, instruments and surfaces in hospital.

Keywords: Copper; Antibacterial activity; Methicillin-resistant Staphylococcus aureus; Vancomycin resistant Enterococcus faecium; Intensive care units 


\section{INTRODUCTION}

Currently healthcare-associated infections (HAIs) are associated with increasing medical costs and significant disability to the patients $[1,2]$. Moreover, those are the causes of prolonged hospitalization periods, and increased mortality and morbidity [3-5]. In intensive care units (ICUs) in particular, aside from disease severity, there are various risk factors for HAIs such as the use of invasive procedures and instruments, use of broad-spectrum antibiotics, and a high level of colonization by pathogenic organisms. In HAIs, the proportion of multidrug-resistant organisms as primary pathogens is increasing annually [6].

Infection-control measures including hand hygiene, active surveillance cultures, and environmental cleaning are generally recommended to reduce the HAIs [7]. It is recognized that hospital instruments are closely associated with the spread of infection as inappropriately sterilized instruments and environmental surface contamination leads to the emergence of infectious disease [8]. Contaminated environmental surfaces by microorganisms do not directly cause infection of patients and medical personnel, but offer a potential chance of spread of infection through surface contact [9]. Hence, the method of cleaning and disinfection must be adequate to counter this threat. Microorganisms will still remain on environmental surfaces because disinfectant efficacy declines as time passes. The methicillin resistant Staphylococcus aureus (MRSA) pathogen, which is a major cause of HAIs, can survive in a non-biosphere such as on the glass or steel from 4 weeks to 7 months, vancomycin resistant Enterococcus faecium (VRE) from 5 days to 4 months, Acinetobacter spp. from 3 days to 5 months, Pseudomonas spp. from 6 hours to 16 months, and Candida spp. from 1 to 150 days [10].

Copper and copper alloys are recognized to have bactericidal and bacteriostatic properties. Previous studies have attempted to verify the antimicrobial activity of copper in different environments [11-13]. It has been shown that copper surfaces have antimicrobial properties and therefore have reduced a numbers of microorganism colonies. This decreases the chances of contamination of medical personnel via fixtures and fittings or medical instruments. The activity of copper in this regard has not been tested in a clinical study. In the present study we assessed the antimicrobial properties of copper and copper alloy against MRSA and VRE and demonstrated the reduction of microbial environmental colonization in an ICU setting of a Korean hospital.

\section{METHODS}

The study was approved by the Institutional Review Board of Asan Medical Center, Seoul, Korea (2011-0466) and was conducted in the laboratory and medical ICU at Asan Medical Center with 18 medical ICU beds for 3 months. MRSA and VRE were supplied by Korean cell culture center (Seoul, Korea), and were incubated in tryptone soy broth at $37^{\circ} \mathrm{C}$ with aeration. Copper, copper alloy, and stainless steel experimental plates $(5 \times 5$ $\mathrm{cm}$, provided by the International Copper Association, Seoul, Korea) were sterilized in an autoclave. After incubation, the plates were inoculated with $40 \mu \mathrm{L}$ of MRSA or VRE containing approximately $6 \times 10^{4}$ colony-forming units $(\mathrm{CFU}) / \mathrm{mL}$ at room temperature $\left(24^{\circ} \mathrm{C}\right)$, and $10.7 \%$ humidity. Using the stamp method, sampling was done at 10 time points $(15,30,45,60,75,90,180$ minutes, 6 , 12, and 24 hours). The plates were incubated at $37^{\circ} \mathrm{C}$ for 48 hours and CFUs were counted. All experiments were performed in triplicate.

\section{In vivo analysis}

Two similar rooms were chosen among the 18 medical ICUs at Asan Medical Center. One room had copper-containing surface items that were touched often by medical personnel. The other room contained stainless steel surface items that included an infusion pole, a bedside rail, a door knob, a drawer and a drug cart. The items of two rooms were the same but the surfaces were covered with different materials, and were sampled once weekly at 9:30 AM after routine cleanings and again at 11:30 AM after visiting hours, during that time patients had received healthcare workers or visitors. A sterile swab moistened in $0.9 \%$ sterile saline was applied five times horizontally and vertically over a $5 \times 5 \mathrm{~cm}$ area. Samplings were transferred to the laboratory and each CFU was counted. The same disinfectant and protocol was used for both the copper and non-copper-containing surface items. Temperature and humidity were also similar for each tested surface. 


\section{Statistical methods}

The median total CFU count was compared that were remained at according to time interval. Using the chisquare test, we evaluated the antimicrobial activity on the tested metal surfaces assuming that a decreased colony count means higher antimicrobial activity. The statistical analysis were performed with IBM SPSS Statistics version 20.0 (IBM Co., Armonk, NY, USA). A p value of $<0.05$ was considered statistically significant

\section{RESULTS}

In vitro time-kill curves were obtained in triplicate and indicated that exposure to copper significantly reduced, and in some cases completely killed MRSA colonies after 15 minutees, which was maintained for 24 hours. On copper alloy plates, MRSA colonies were decreased by nearly 90\% after 15 minutes and completely killed at 12 hours, and this was maintained for 24 hours. In comparison, MRSA colonies only slowly decreased on stainless steel plates and $5 \%$ of the colonies remained after 24 hours (Fig. 1).

On copper plates, the time-kill curves for VRE were similar to those for MRSA. VRE colonies were found to be significantly reduced and fully eradicated at 15 minutes, which was maintained for 24 hours. On copper alloy plates, $3 \%$ of the VRE colonies remained at 15 minutes, VRE colonies were completely destroyed af-

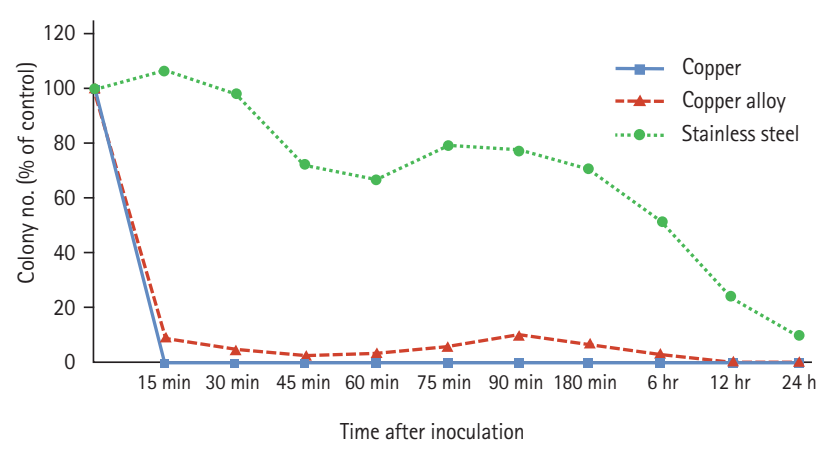

Figure 1. Time-kill study of methicillin resistant Staphylococcus aureus on copper surfaces compared with controls: colony-forming unit number (\%) vs. time after inoculation. Data represent mean \pm standard deviation of triplicate measurements. ter 12 hours, and this was maintained for 24 hours. In comparison, on stainless steel plates, VRE colonies were only slightly decreased as time progressed and $45 \%$ of the colonies persisted (Fig. 2).

The median CFU count per plate from our in vivo analysis of MRSA, comparing copper-containing surfaces with non-copper-containing surfaces is summarized in Table 1. Compared with the controls, MRSA colonies were reduced after cleaning and after patients were seen by their visitors in the copper-containing room. At 9:30 AM, after cleaning, the measured bacterial contamination rate low with statistical significance at the infusion pole, bedside rail, door, and drawer (Fig. 3A). At 11:30 AM, after patients were seen by their visitors, the contamination rate was low only at the door (Fig. 3B). Fig. 3 shows our quantitative analysis of MRSA on copper-containing items compared with the controls.

Similar to MRSA, the contamination rate of VRE was low on copper-containing items compared with the controls. VRE was low when measured at the infusion pole after cleaning of the room (Fig. 4) and at the door after patients were seen by their visitors. This was found to be statistically significant and is shown in Table 2.

Hospital equipments (such as infusion pole, a bedside rail, a door knob, a drawer, and a drug cart) in the ICU were used in the experiments (Supplementary Fig. 1).

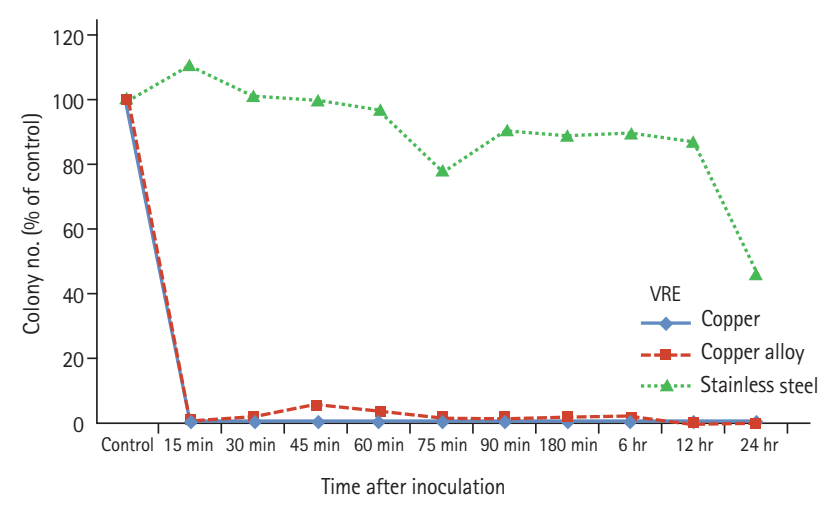

Figure 2. Time-kill study of vancomycin resistant Enterococcus faecium (VRE) on copper surfaces compared with controls: colony-forming unit number (\%) vs. time after inoculation. Data represent mean \pm standard deviation of triplicate measurements. 
Table 1.Quantitative analysis of methicillin resistant Staphylococcus aureus on copper-containing items compared with controls

\begin{tabular}{|c|c|c|c|c|c|c|c|}
\hline \multirow[t]{2}{*}{ Item } & \multirow{2}{*}{$\begin{array}{c}\text { Time sampled, } \\
\text { AM }\end{array}$} & \multicolumn{2}{|c|}{$\begin{array}{l}\text { Colony forming } \\
\text { unit count per plate }\end{array}$} & \multirow[t]{2}{*}{$p$ value } & \multicolumn{2}{|c|}{$\begin{array}{l}\text { Mean colony forming } \\
\text { unit count per plate }\end{array}$} & \multirow[t]{2}{*}{$p$ value } \\
\hline & & Control & Copper & & Control & Copper & \\
\hline \multirow[t]{2}{*}{ Infusion pole } & $9: 30$ & $1(0-4)$ & $0(0-0)$ & $0.001^{a}$ & 4.7 & 0.3 & $0.05^{b}$ \\
\hline & $11: 30$ & $0(0-2)$ & $0(0-0)$ & $0.04^{b}$ & 2.6 & 0.3 & $0.05^{b}$ \\
\hline \multirow[t]{2}{*}{ Bedside rail } & $9: 30$ & $1(0-10)$ & $\circ(0-1)$ & $0.001^{a}$ & 12.5 & 3.7 & $0.04^{b}$ \\
\hline & $11: 30$ & $2(0-9)$ & $0(0-6)$ & 0.15 & 11.8 & $13 \cdot 3$ & 0.87 \\
\hline \multirow[t]{2}{*}{ Door } & $9: 30$ & $2(0-4)$ & $0(0-0)$ & $<0.001^{\mathrm{a}}$ & 2.2 & 0.7 & $0.02^{b}$ \\
\hline & $11: 30$ & $1(0-3)$ & $0(0-0)$ & $<0.001^{\mathrm{a}}$ & $7 \cdot 4$ & 0.4 & $0.02^{b}$ \\
\hline \multirow[t]{2}{*}{ Drawer } & $9: 30$ & $0(0-1)$ & $0(0-0)$ & $0.01^{b}$ & 5.6 & 1.2 & 0.14 \\
\hline & $11: 30$ & $0(0-2)$ & $0(0-0)$ & 0.14 & 2.0 & 0.4 & 0.22 \\
\hline \multirow[t]{2}{*}{ Drug cart } & $9: 30$ & $3(1-8)$ & $1(0-4)$ & 0.11 & 12.1 & 4.8 & 0.22 \\
\hline & $11: 30$ & $4(1-12)$ & $1(0-4)$ & $0.02^{b}$ & 11.0 & 5.7 & $0.05^{b}$ \\
\hline
\end{tabular}

Values are presented as median (interquartile range).

$\mathrm{a} p \leq 0.001$.

$\mathrm{b} p \leq 0.05$.
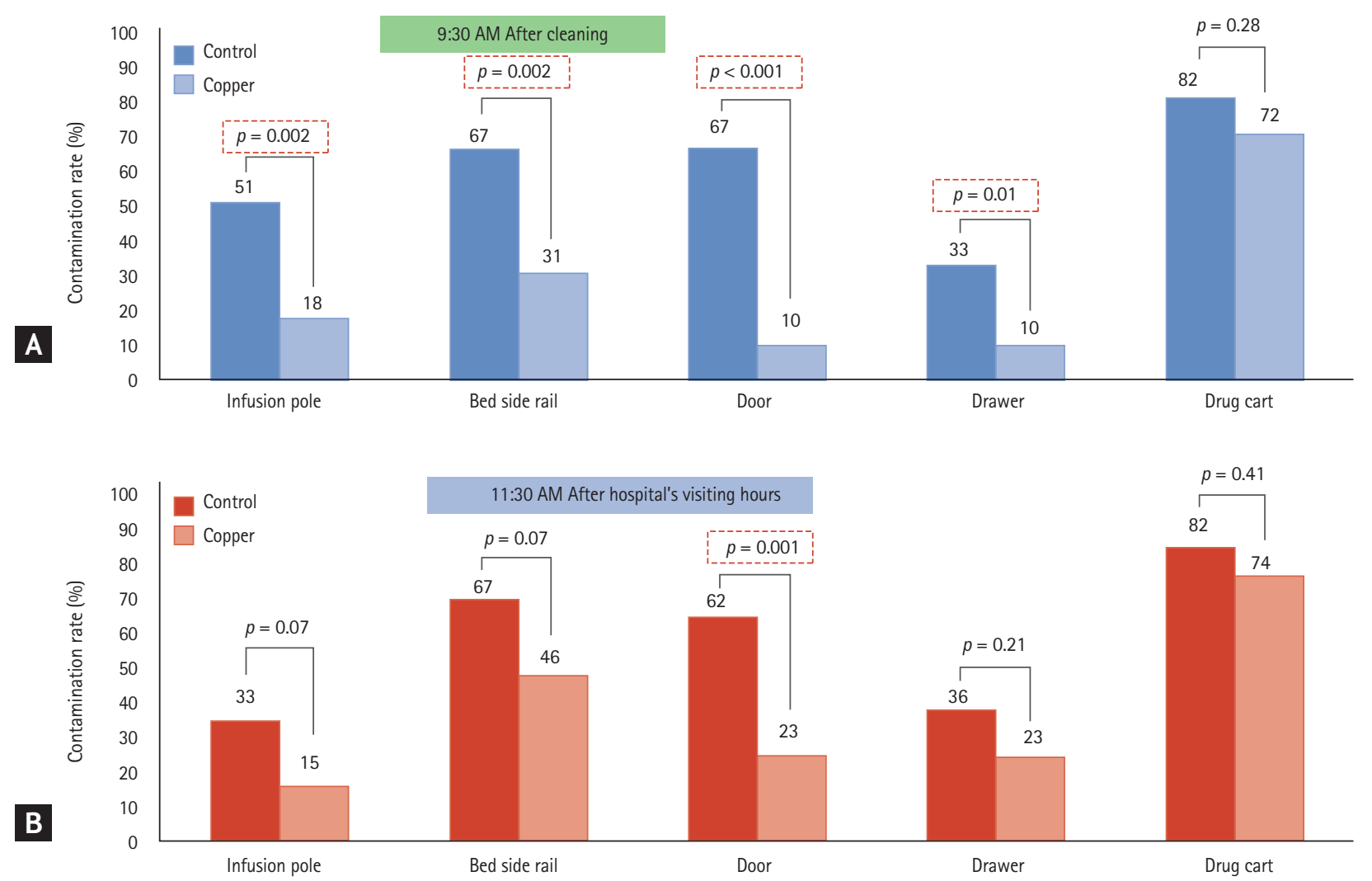

Figure 3. Contamination rate of methicillin resistant Staphylococcus aureus according to the hospital equipments at (A) 9:30 AM after cleaning and (B) 11:30 AM after hospital visiting hours. All experiments were performed in triplicate (mean \pm standard deviation). 


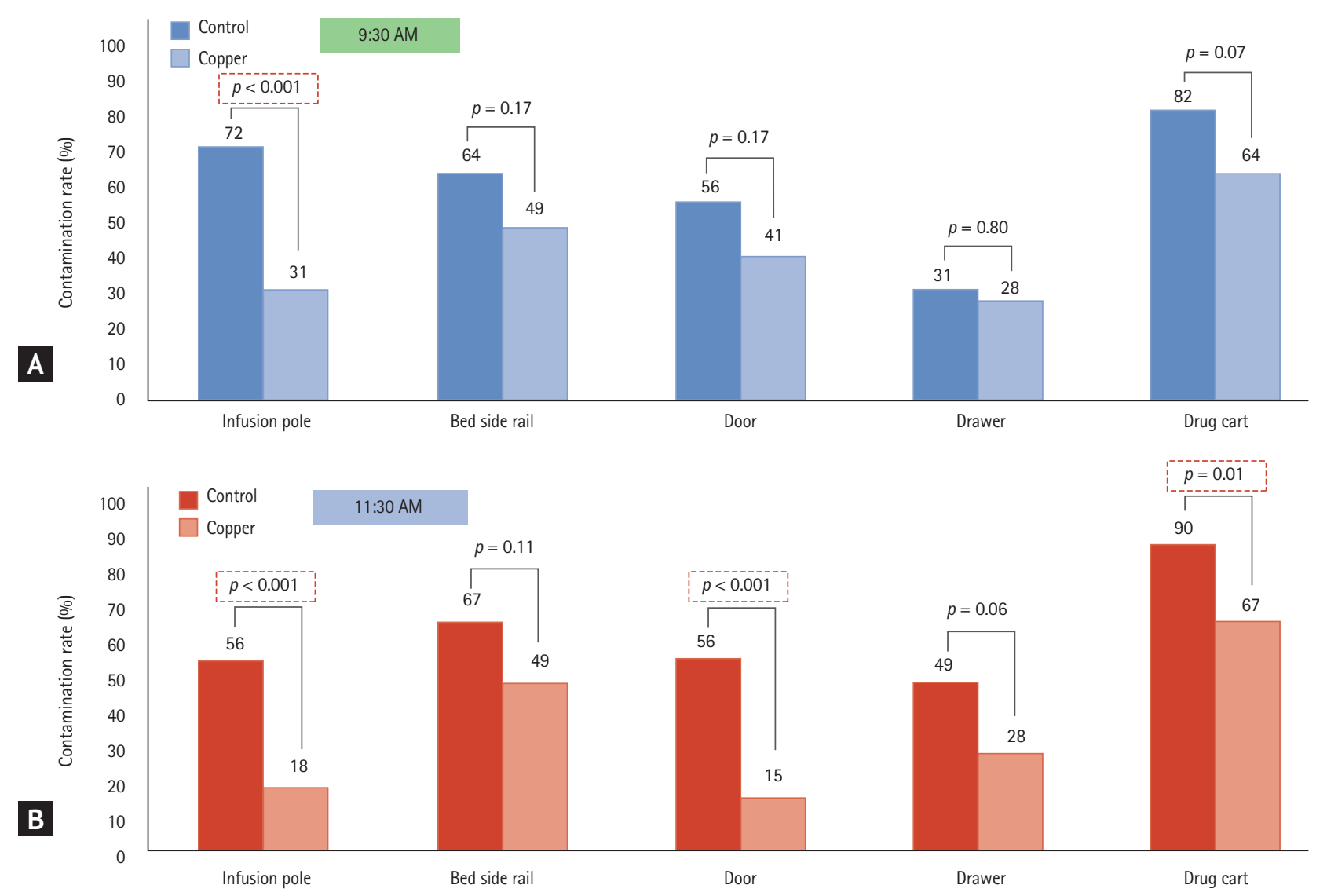

Figure 4. Contamination rate of vancomycin resistant Enterococcus faecium according to the hospital equipments at (A) 9:30 AM after cleaning and (B) 11:30 AM after hospital visiting hours. All experiments were performed in triplicate (mean \pm standard deviation).

Table 2. Quantitative analysis of vancomycin resistant Enterococcus faecium on copper-containing items compared with controls

\begin{tabular}{|c|c|c|c|c|c|c|c|}
\hline \multirow[t]{2}{*}{ Item } & \multirow{2}{*}{$\begin{array}{c}\text { Time } \\
\text { sampled, AM }\end{array}$} & \multicolumn{2}{|c|}{$\begin{array}{l}\text { Colony forming } \\
\text { unit count per plate }\end{array}$} & \multirow[t]{2}{*}{$p$ value } & \multicolumn{2}{|c|}{$\begin{array}{l}\text { Mean colony forming } \\
\text { unit count per plate }\end{array}$} & \multirow[t]{2}{*}{$p$ value } \\
\hline & & Control & Copper & & Control & Copper & \\
\hline \multirow[t]{2}{*}{ Infusion pole } & 9:30 & $2(0-4)$ & $\mathrm{o}(0-1)$ & $0.001^{a}$ & $4 \cdot 3$ & 0.7 & $0.006^{\mathrm{b}}$ \\
\hline & 11:30 & $1(0-3)$ & $0(0-0)$ & $0.001^{a}$ & 4.2 & 0.4 & $0.007^{\mathrm{b}}$ \\
\hline \multirow[t]{2}{*}{ Bed side rail } & $9: 30$ & $1(0-4)$ & $\circ(0-2)$ & 0.10 & 8.2 & 2.1 & 0.13 \\
\hline & 11:30 & $2(0-5)$ & $0(0-3)$ & 0.07 & 8.8 & 4.9 & 0.32 \\
\hline \multirow[t]{2}{*}{ Door } & $9: 30$ & $1(0-3)$ & $0(0-1)$ & 0.06 & 3.6 & 2.4 & 0.54 \\
\hline & 11:30 & $1(0-2)$ & $0(0-0)$ & $<0.001$ & 1.7 & 0.5 & $0.02^{b}$ \\
\hline \multirow[t]{2}{*}{ Drawer } & $9: 30$ & $O(0-1)$ & $o(0-1)$ & 0.84 & 0.8 & 1.1 & 0.72 \\
\hline & 11:30 & $0(0-2)$ & $0(0-1)$ & 0.12 & 6.2 & 6.5 & 0.96 \\
\hline \multirow[t]{2}{*}{ Drug cart } & $9: 30$ & $3(1-5)$ & $1(0-3)$ & $0.04^{b}$ & 3.6 & 2.5 & 0.21 \\
\hline & 11:30 & $3(1-5)$ & $1(0-3)$ & $0.003^{b}$ & 4.7 & 2.1 & $0.01^{b}$ \\
\hline
\end{tabular}

Values are presented as median (interquartile range).

${ }^{a} p \leq 0.001$.

$\mathrm{b} p \leq 0.05$. 


\section{DISCUSSION}

In this study, we investigated contact killing effect of copper and copper alloy surface in vitro and we demonstrated the reduction on microbial colonization on the copper surface in vivo hospital setting, especially in ICU.

There have been many attempts to reduce HAI rates in different ways since the emergence of multi-drug resistant organisms and the increased number of infections by them. It is well known that copper and copper alloy have bactericidal and bacteriostatic effect [11-13].

Still there are several studies of copper alloys on the gram negative bacterial infections. Souli et al. [12] investigated antimicrobial activity of copper surfaces against carbapenemase-producing contemporary Gram-negative clinical isolates: Escherichia coli, Enterobacter spp., Klebsiella pneumoniae, Pseudomonas aeruginosa, and Acinetobacter baumannii producing either VIM-1 and/or KPC2 or VIM-2 or OXA-type carbapenemases. Eser et al. [14] reported antimicrobial activity of copper alloys against invasive multidrug-resistant nosocomial pathogens. Clinical isolates of MRSA ( $\mathrm{n}=4)$, MDR Acinetobacter baumannii $(\mathrm{n}=6)$, and Pseudomonas aeruginosa $(\mathrm{n}=4)$ were evaluated. Cug9 showed a bactericidal effect of less than 1 hour for MRSA, 2 hours for P. aeruginosa and exhibited a closer bactericidal range effect against $A$. baumannii. Rozanska et al. [15] showed antimicrobial effect of copper alloys on Acinetobacter species isolated from infections and hospital environment. And the results of the study confirmed effective activity (bacteriocidal or bacteriostatic) of copper alloys [15].

Further studies will warrant elucidating the clinical application of our present experimental results that copper alloys reduce MRSA or VRE infection; that is to say, diminishing transmission of infections by copper alloys or decreased number of HAI by those.

To take advantage of copper's antimicrobial activity, many clinical studies have tried to utilize copper surfaces and instruments in the hospital environment for the purpose of reducing the microbial burden and hospital associated infections [16-19]. We have here demonstrated that copper and copper alloys suppress the growth of multi-drug resistant microorganisms and exhibits a killing effect on these microbes.

In addition we observed that copper can suppress multi-drug resistant bacterias (MRSA and VRE) and eliminate them. The mechanistic action of copper on bacteria has been determined previously as copper ions can bind or cross-link DNA strands and damage DNA [20].

The future studies need to be focus on other types of multi-drug resistant organisms as we are not able to generalize our present experimental results to other strains. The cost- effectiveness of widespread copper use is an issue as it is an expensive metal. Moreover, copper is easy to use but can accumulate rust over time. We thus need to consider how to preserve the properties of copper whilst preventing rust. And copper is able to conduct electricity, that astonishes very transiently the person who touches copper.

This study is to observe the antimicrobial activity of copper in a Korean hospital environment. Although proper hand hygiene remains a more important barrier against HAIs than antimicrobial surfaces [13], potential of copper as another barrier is warranted.

\section{KEY MESSAGE}

1. Copper and copper alloys suppress multidrug resistant bacteria (methicillin resistant Staphylococcus aureus and vancomycin resistant Enterococcus faecium) and eliminate them on the surfaces of material in vitro.

2. The microbial colonization is reduced on the copper surfaces in vivo hospital setting, especially in intensive care unit.

\section{Conflict of interest}

This study was supported by the International Copper Association, Seoul, Korea with assistance from Poongsan, LS Nikko.

\section{REFERENCES}

1. Roberts RR, Scott RD 2nd, Hota B, et al. Costs attributable to healthcare-acquired infection in hospitalized adults and a comparison of economic methods. Med Care 2010;48:1026-1035.

2. Haque M, Sartelli M, McKimm J, Abu Bakar M. Health care-associated infections: an overview. Infect Drug Resist 
2018;11:2321-2333.

3. Cosgrove SE. The relationship between antimicrobial resistance and patient outcomes: mortality, length of hospital stay, and health care costs. Clin Infect Dis 2006;42 Suppl 2:S82-S89.

4. Graves N, Weinhold D, Tong E, et al. Effect of healthcare-acquired infection on length of hospital stay and cost. Infect Control Hosp Epidemiol 2007;28:280-292.

5. De Angelis G, Murthy A, Beyersmann J, Harbarth S. Estimating the impact of healthcare-associated infections on length of stay and costs. Clin Microbiol Infect 2010;16:1729-1735.

6. Weiner LM, Webb AK, Limbago B, et al. Antimicrobial-resistant pathogens associated with healthcare-associated infections: summary of data reported to the national healthcare safety network at the centers for disease control and prevention, 2011-2014. Infect Control Hosp Epidemiol 2016;37:1288-1301.

7. O'Grady NP, Alexander M, Burns LA, et al. Guidelines for the prevention of intravascular catheter-related infections. Clin Infect Dis 2011;52:e162-e193.

8. Boyce JM. Environmental contamination makes an important contribution to hospital infection. J Hosp Infect 2007;65 Suppl 2:50-54.

9. Otter JA, Yezli S, Salkeld JA, French GL. Evidence that contaminated surfaces contribute to the transmission of hospital pathogens and an overview of strategies to address contaminated surfaces in hospital settings. Am J Infect Control 2013;41:S6-S11.

10. Kampf G, Kramer A. Epidemiologic background of hand hygiene and evaluation of the most important agents for scrubs and rubs. Clin Microbiol Rev 2004;17:863-893.

11. Noyce JO, Michels H, Keevil CW. Potential use of copper surfaces to reduce survival of epidemic meticillin-resistant Staphylococcus aureus in the healthcare environment. J Hosp Infect 2006;63:289-297.
12. Souli M, Galani I, Plachouras D, et al. Antimicrobial activity of copper surfaces against carbapenemase-producing contemporary Gram-negative clinical isolates. J Antimicrob Chemother 2013;68:852-857.

13. Noyce JO, Michels H, Keevil CW. Use of copper cast alloys to control Escherichia coli O157 cross-contamination during food processing. Appl Environ Microbiol 2006;72:4239-4244.

14. Eser OK, Ergin A, Hascelik G. Antimicrobial activity of copper alloys against invasive multidrug-resistant nosocomial pathogens. Curr Microbiol 2015;71:291-295.

15. Rozanska A, Chmielarczyk A, Romaniszyn D, Majka G, Bulanda M. Antimicrobial effect of copper alloys on Acinetobacter species isolated from infections and hospital environment. Antimicrob Resist Infect Control 2018;7:10.

16. Casey AL, Adams D, Karpanen TJ, et al. Role of copper in reducing hospital environment contamination. J Hosp Infect 2010;74:72-77.

17. Karpanen TJ, Casey AL, Lambert PA, et al. The antimicrobial efficacy of copper alloy furnishing in the clinical environment: a crossover study. Infect Control Hosp Epidemiol 2012;33:3-9.

18. Salgado CD, Sepkowitz KA, John JF, et al. Copper surfaces reduce the rate of healthcare-acquired infections in the intensive care unit. Infect Control Hosp Epidemiol 2013;34:479-486.

19. Sifri CD, Burke GH, Enfield KB. Reduced health care-associated infections in an acute care community hospital using a combination of self-disinfecting copper-impregnated composite hard surfaces and linens. Am J Infect Control 2016;44:1565-1571.

20. Weaver L, Noyce JO, Michels HT, Keevil CW. Potential action of copper surfaces on meticillin-resistant Staphylococcus aureus. J Appl Microbiol 2010;109:2200-2205. 


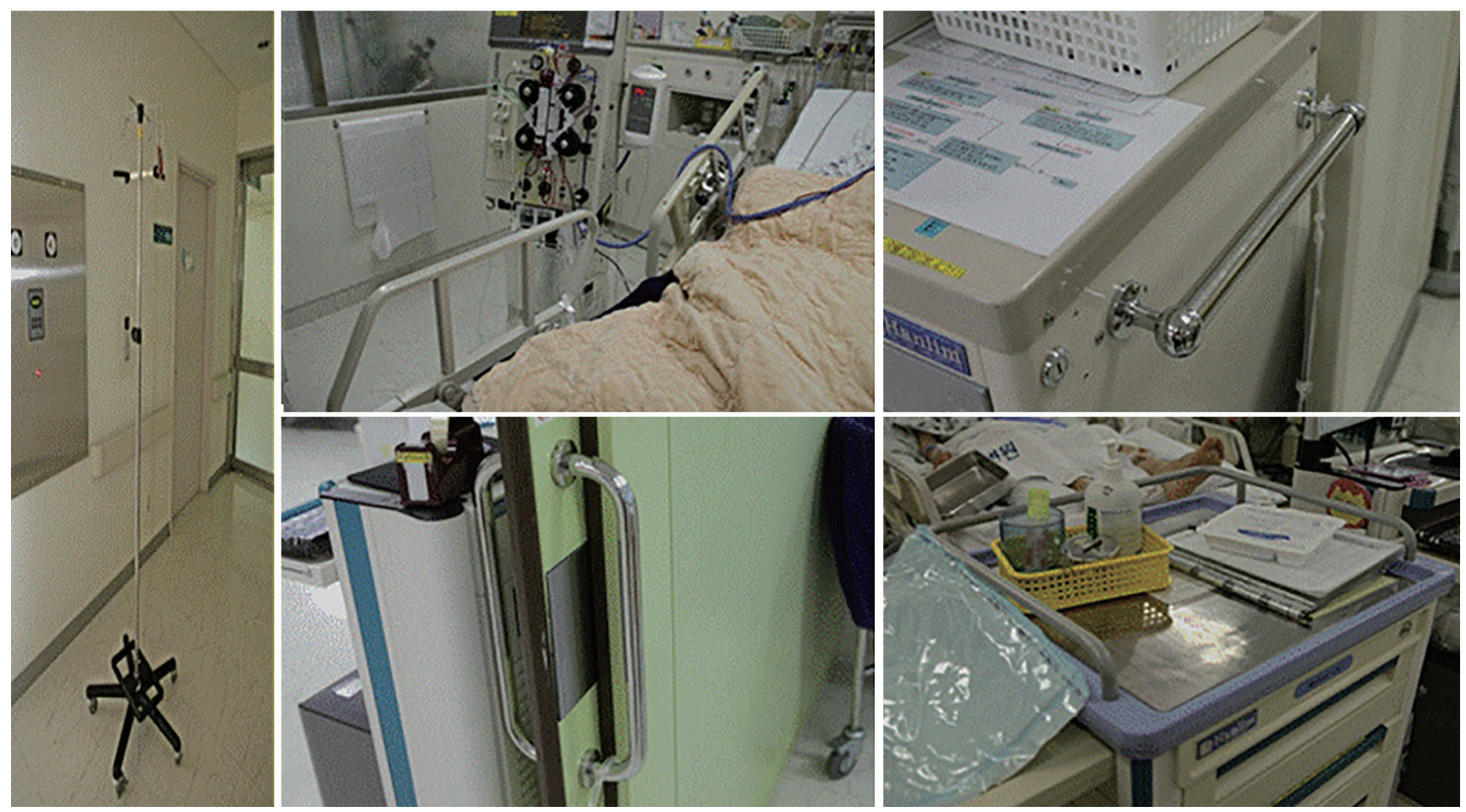

Supplementary Figure 1. Hospital equipments (such as infusion pole, a bedside rail, a door knob, a drawer, and a drug cart) in the intensive care unit were used in the experiments. 